\title{
Catarata pediátrica en un país en vías de desarrollo: revisión retrospectiva de 328 casos
}

\author{
Pediatric cataract in a developing country: retrospective review of 328 cases
}

\author{
Martin A. Zimmermann-Paiz ${ }^{1}$, Carlos R. Quiroga-Reyes ${ }^{1}$
}

\section{RESUMEN}

Introducción: La catarata en edad pediátrica constituye una causa importante de disminución de la agudeza visual. El objetivo del presente trabajo es conocer las características de esta patología en una unidad de atención ocular de un país en vías de desarrollo.

Métodos: Se realizó un estudio retrospectivo, descriptivo, transversal de 328 casos de catarata en edad pediátrica (niños entre 0 y 14 años).

Resultados: Se encontró 190 (57.9\%) casos de sexo masculino y 138 (42.1\%) femenino, 145 (44.2\%) casos de catarata congénita y 183 (55.8\%) de adquirida. 160 (48.8\%) presentaron afección unilateral y 168 (51.2\%) bilateral. El tratamiento fue quirúrgico en $98.2 \%$ de los casos. La edad media de diagnóstico en las cataratas congénitas fue 34.9 meses. La edad media de presentación en las adquiridas fue 76.6 meses. Se encontró abandono de tratamiento en $70.58 \%$ de cataratas congénitas y $64.1 \%$ de adquiridas. En las congénitas el 44\% presentó agudeza visual en el rango de 20/200 a 20/70 y en las adquiridas el $32.5 \%$ mayor a 20/30

Conclusiones: Se encontró datos poco alentadores como la detección tardía y el abandono del seguimiento, los cuales son factores de mal pronóstico.

Descriptores: Enfermedades de los ojos; Catarata; Niño; País en desarrollo

\begin{abstract}
Introduction: Pediatric cataracts constitute an important cause of low visual acuity. Purpose: The objective of the present work is to know the characteristics of this pathology in an ophthalmic attention unit of a developing country.

Methods: A retrospective, descriptive, transversal study was carried out in 328 cases of pediatric cataracts (children between 0 and 14 years).

Results: 190 (57.9\%) patients were male and 138 (42.1\%) female, 145 (44.2\%) cases were congenital cataracts and 183 (55.8\%) acquired cataracts; 160 (48.8\%) presented unilateral affection and 168 (51.2\%) bilateral. The treatment was surgical in $98.2 \%$ of the cases. The mean age at diagnosis of congenital cataract was 34.9 months. The mean age of presentation of the acquired ones was 76.6 months. The treatment was abandoned in $70.58 \%$ cases of congenital cataracts and $64.10 \%$ of acquired cataracts. In the congenital ones, 44\% presented visual acuity in the range of 20/200 and 20/70 and in the acquired ones, 32.5\% were better than 20/30.
\end{abstract}

Conclusions: The data found was not encouraging due to the late detection and the abandon of the treatment, which are bad prognosis factors.

Keywords: Eye diseases; Cataracts; Child; Developing countries

\section{INTRODUCCIÓN}

La catarata (Figura 1) se define como una opacidad del cristalino. En la edad pediátrica constituye una causa importante de disminución de la agudeza visual. En la actualidad se estima una incidencia de 1 a 13.6 por 10,000 nacimientos ${ }^{(1-4)}$. En países en desarrollo la tasa de ceguera puede ser tan alta como 1.5 por 1000 habitantes, y la relacionada a la catarata pediátrica representa un enorme problema con relación a morbilidad, pérdida económica y aislamiento social(5). Debido a la expectativa de vida de la población pediátrica, restablecer la visión en un niño ciego por catarata equivaldría a hacerlo en 10 pacientes adultos ${ }^{(6)}$. Con el objeto de conocer las características de esta patología en una unidad de atención ocular de un país en vías de desarrollo, en el presente trabajo se hace una revisión retrospectiva de 328 casos atendidos en un período de 7 años.

\section{METODOS}

Se realizó un estudio retrospectivo, descriptivo, transversal en una serie de 328 casos de catarata en edad pediátrica (se incluyó cataratas congénitas y adquiridas en niños con edad comprendida entre 0 y 14 años) atendidos en el período comprendido de 2000 a 2007 en la Clínica de oftalmología pediátrica estrabismo y neurooftalmología "Dra. Amaría Illescas Putzeys", Hospital de ojos y oídos "Dr. Rodolfo Robles V". Instituto de ciencias de la visión, Benemérito Comité pro Ciegos y Sordos de Guatemala.

Se revisó sistemáticamente cada expediente, y en cada caso se recaudaron los siguientes datos: edad de presentación, edad del diagnóstico, sexo, etnia (mestizo, indígena, otro), procedencia (capital, interior de la república), etiología (Congénitas: idiopática, hereditaria, infecciosa, otros; Adquiridas: traumática, metabólica, imflamatoria, del desarrollo, otros), afectación uni o bilateral, anomalías asociadas (microftalmos, glaucoma, vítreo primario persistente, otros), presencia de signos de mal pronóstico en las cataratas congénitas (nistagmus, estrabismo, unilateral, otros). Abandono o no del tratamiento en los casos con un seguimiento potencial mínimo de 4 años (abandono se definió como la no asistencia a la consulta durante 3 años consecutivos). Agudeza visual en la última evaluación (se utiliza la notación de Snellen) en los casos que no abandonaron el tratamiento (en casos bilaterales se documentó la del
Submitted for publication: September 10,2010 Accepted for publication: April 17, 2011

Study carried out at the Pediatric Ophthalmology, Strabismus and Neuro-Ophthalmology Clinic "Dra. Amaría Illescas Putzeys", Eye and Ear Hospital "Dr. Rodolfo Robles V." Vision Science Institute, Benemérito Comité pro Ciegos y Sordos de Guatemala - Guatemala.

Clínica de Oftalmología Pediátrica Estrabismo y Neuro-oftalmología "Dra. Amaría Illescas Putzeys", Hospital de ojos y oídos "Dr. Rodolfo Robles V." Instituto de Ciencias de la Visión, Benemérito Comité pro Ciegos y Sordos de Guatemala - Guatemala.
Funding: No specific financial support was available for this study.

Disclosure of potential conflicts of interest: M.A.Zimmermann-Paiz, None; C.R.Quiroga-Reyes, None.

Correspondence address: Martin A. Zimmermann-Paiz. Diagonal 21, 19-19 Anillo Periférico Zona 11 Guatemala - Guatemala C.A. - E-mail: oftalmopedia@gmail.com 

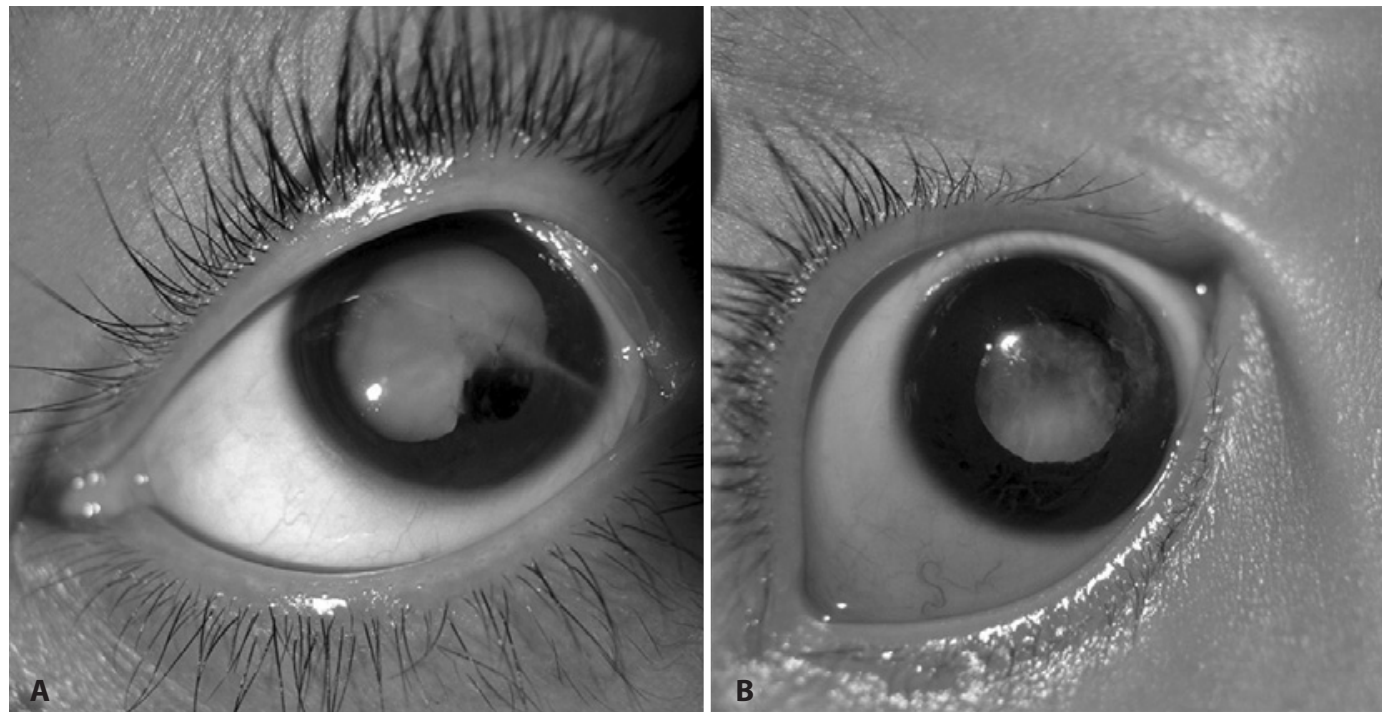

Figura 1. A) Catarata adquirida en el ojo izquierdo de un niño de 7 años. La causa fue un trauma penetrante. Nótese la opacidad lineal en la cornea secundaria a la herida; B) Catarata congénita bilateral (se muestra el ojo derecho) de un niño de 9 meses de edad. La causa fue desconocida.

mejor ojo). Para el análisis estadístico, los datos se consignaron a una hoja electrónica diseñada para el efecto utilizando el programa EPI-INFO (Database and stadistics software for public health professionals, Centers for Disease Control and Prevention 2008).

\section{RESULTADOS}

Se estudiaron 328 casos, 190 (57.9\%) de sexo masculino y 138 (42.1\%) femenino. De los pacientes estudiados 270 (82.3\%) se catalogaron como mestizos, 40 (12.2\%) indígenas y 18 (5.5\%) con etnia desconocida. Con respecto a la procedencia 113 (34.5\%) casos fueron originarios de la ciudad capital y 215 (65.5\%) del área rural de la república. En cuanto a la etiología se encontró 145 (44.2\%) casos de catarata congénita y 183 (55.8\%) de adquirida. En la tabla 1 se detalla lo encontrado respecto a la etiología. Del total de casos 160 (48.8\%) presentaron afección unilateral y 168 (51.2\%) bilateral. El tratamiento fue quirúrgico en 322 (98.2\%) de los casos y conservador en $6(1.8 \%)$

Con respecto al sub grupo de cataratas congénitas se encontró: 117 (80.7\%) casos con afección bilateral y 28 (19.3\%) unilateral. 11 casos (7.6\%) presentaron alguna anomalía ocular asociada. En 74 (51\%) casos se documentó uno o más signos clínicos de mal pronóstico (afección unilateral, nistagmos, estrabismo). La edad media de diagnóstico en las cataratas congénitas fue 34.9 meses (Desviación estándar DS: 41.74). El 10.6\% de los pacientes con catarata congénita fueron diagnosticados en los primeros 2 meses de vida, el 40.8\% entre los 3 a 12 meses y el resto (48.6\%) luego del primer año. En 141 (97.2\%) de los casos el tratamiento fue quirúrgico. En 51 (35.2\%) de los casos de catarata congénita se realizo implante de lente intraocular. Se identificó que 85 (58.62\%) casos de catarata congénita tuvieron potencialmente entre 4 y 7 años de seguimiento. De estos, 60 (70.58\%) casos se catalogaron como abandono de tratamiento. En la tabla 2 se puede observar lo encontrado con respecto a la agudeza visual del mejor ojo en la última consulta registrada.

Con respecto al sub grupo de catarata adquirida se encontró: La edad media de presentación en las cataratas adquiridas fue 76.6 meses (DS: 37.7 ), y la edad media de diagnóstico 84.5 meses (DS: 36.6). El $42.6 \%$ de las cataratas adquiridas se presentaron en los primeros 5 años (60 meses) de vida, 45.4\% en los segundos 5 años (61 a 120 meses) de vida y el resto en los siguientes 5 años (121 a
Tabla 1. Etiologia encontrada según cada subgrupo

\begin{tabular}{lrlr}
\hline & $\mathbf{N}(\%)$ & & $\mathbf{N}(\%)$ \\
Congénita & $\mathbf{1 4 5}(\mathbf{4 4 . 2 )}$ & Adquirida & $\mathbf{1 8 3}(\mathbf{5 5 . 8})$ \\
\hline Causa no determinada & $127(87.6)$ & Traumática & $89(48.6)$ \\
Infecciosa & $11(7.6)$ & Causa no determinada & $47(25.7)$ \\
Hereditaria & $0(0)$ & Catarata del desarrollo & $29(15.8)$ \\
Sindromes & $6(4.1)$ & Imflamatoria & $16(8.7)$ \\
Otros & $1(0.7)$ & Metabolica & $0(0)$ \\
& & Otros & $2(1.1)$ \\
\hline
\end{tabular}

Tabla 2. Agudeza visual según cada subgrupo

\begin{tabular}{lcc}
\hline $\begin{array}{l}\text { Rango de agudeza visual } \\
\text { (notación de Snellen) }\end{array}$ & $\begin{array}{c}\text { Catarata congénita } \\
\text { N (\%) }\end{array}$ & $\begin{array}{c}\text { Catarata adquirida } \\
\text { N (\%) }\end{array}$ \\
\hline Menor a 20/200 & $7(28.0)$ & $35(29.9)$ \\
$20 / 200$ a 20/70 & $11(44.0)$ & $20(17.1)$ \\
$20 / 60$ a 20/30 & $1(4.0)$ & $19(16.2)$ \\
Mayor a 20/30 & $0(0)$ & $38(32.5)$ \\
No cuantificable & $6(24.0)$ & $5(4.3)$ \\
\hline
\end{tabular}

180 meses). Se encontró 131 (71.6\%) casos de afección unilateral y 52 (28.4\%) bilateral. En 181 (98.9\%) casos el tratamiento fue quirúrgico. En 133 casos (72.7\%) se realizo implante de lente intra ocular. Se identificó 117 (63.9\%) casos de catarata adquirida tuvieron potencialmente entre 4 y 7 años de seguimiento. De estos, 75 (64.1\%) casos se catalogaron como abandono de tratamiento. En la tabla 2 se puede observar lo encontrado con respecto a la agudeza visual del mejor ojo en la última consulta registrada.

\section{DISCUSIÓN}

El desarrollo visual tiene un período crítico, durante el cual cualquier insulto que provoque una alteración del estimulo visual normal (uni o bilateral), tendrá como resultado el aparecimiento de ambliopía. El período más sensible se presenta en los primeros 3 años de vida ${ }^{(7)}$, pero puede extenderse. Por lo tanto el propósito 
principal del diagnóstico precoz de la catarata es evitar las complicaciones irreversibles, principalmente la ambliopía, que influye directamente en el pronóstico visual del niño. En el Continente Americano se estima unos 100,000 niños $\operatorname{ciegos}^{(8)}$, con un porcentaje de ceguera secundaria a problemas del cristalino de un $7 \%{ }^{(9)}$, por lo que constituye una causa de ceguera prevenible muy importante. La presente serie mostró que en nuestro medio, la catarata se presenta en similar proporción tanto en etiología congénita como adquirida, datos que difieren de reportes similares en países industrializados ${ }^{(10,11)}$, en donde predomina la causa congénita y se presenta con poca frecuencia, especialmente en niños con retardo del crecimiento intrauterino ${ }^{(12)}$. En nuestro medio no tenemos datos sobre la prevalencia de esta patología, pero sospechamos que es alta. Los datos encontrados mostraron que en la mayoría de casos de catarata congénita no se identifico la causa, dato similar al encontrado en otros reportes ${ }^{(10,11,13,14)}$. En estos pacientes podría ser importantes insultos hacia el feto no determinados como toxinas, infecciones, abortivos, factores ambientales, factores genéticos y en nuestro medio en particular, casos en los que no se logra estudiar al paciente por múltiples factores. Contrario a estos informes ${ }^{(10,11,13,14)}$, nuestros datos no mostraron casos de cataratas hereditarias, a pesar que principalmente se trataron de casos con problema bilateral. Este dato parece interesante, pero podría tratarse simplemente de ausencia de estos datos debido a la naturaleza retrospectiva del trabajo. La presente serie mostro una edad promedio de diagnóstico en las cataratas congénitas sumamente elevada, solo $10.6 \%$ se diagnosticaron en los primeros 2 meses de vida lo que representa un factor de mal pronóstico visual. En nuestro medio existe una gran cantidad de partos extra hospitalarios, lo cual limita probablemente la detección temprana; aunado a esto una buena cantidad de casos provienen del interior de la república, en donde el acceso a cuidados de salud es más limitada. Por factores que desconocemos el abandono de seguimiento es elevado, siendo otro factor de mal pronóstico debido a la importancia que tiene la identificación y tratamiento de la ambliopía. En los pacientes con catarata congénita que no abandonaron el seguimiento, la agudeza visual en el mejor ojo se presentó principalmente en el rango de 20/200 a 20/70, el cual corresponde a un impedimento visual moderado. Este dato es similar al de otros reportes ${ }^{(15,16)}$, pero solo son los datos de los pacientes que no abandonaron el seguimiento. Desconocemos que factores estén implicados de manera directa con este resultado. En cuanto a las cataratas adquiridas, la presente serie mostró que la principal causa es el trauma, lo cual concuerda con otros reportes ${ }^{(10)}$. No se estudió el tipo de trauma ni el lugar en donde ocurrió, interrogantes que implican futuras investigaciones que den información útil para tomar medidas preventivas. Se encontró un porcentaje de casos en el cuál no se identificó la causa. Entre estos pacientes probablemente están incluidos traumatismos no identificados, cataratas del desarrollo, problemas inflamatorios y otros en donde no se encontró una historia clara o evidencias del causante. El 88\% de los casos de catarata adquirida se presentó en los primeros 10 años de vida, con un promedio de 6.4 años al diagnóstico y tiempo promedio de retardo en la consulta de 7.9 meses. Estos pacientes están en riesgo de ambliopía tanto por la edad como por el retardo en la consulta. De manera similar a la catarata congénita, por causas que desconocemos también se observó un alto porcentaje de abandono del seguimiento. En estos pacientes se encontró mejor pronóstico visual, con agudeza visual que está entre impedimento visual leve y rango normal de visión (pacientes que no abandonaron el seguimiento).

Para terminar, meditamos en el hecho de encontrar características poco alentadoras como la detección tardía y el abandono del seguimiento, los cuales consideramos que son factores de mal pronóstico que deben tomarse en cuenta. De los presentes datos surge el reto establecer estrategias para la educación de la población y creación de programas de detección temprana como los recomendados en otros países ${ }^{(17)}$. Concordamos con otros autores ${ }^{(18)}$ en cuanto a que promover la consulta temprana incidirá en el mejoramiento del pronóstico visual, especialmente en las cataratas congénitas.

\section{REFERENCIAS}

1. Zetterström C, Kugelberg M. Paediatric cataract surgery. Acta Ophthalmol Scand. 2007; 85(7):698-710.

2. Lloyd IC, Ashworth J, Biswas S, Abadi RV. Advances in the management of congenital and infantile cataract. Eye (Lond). 2007;21(10):1301-9.

3. Fan DS, Yip WW, Yu CB, Rao SK, Lam DS. Updates on the surgical management of paediatric cataract with primary intraocular lens implantation. Ann Acad Med Singapore. 2006:35(8):564-70.

4. SanGiovanni JP, Chew EY, Reed GF, Remaley NA, Bateman JB, Sugimoto TA, KlebanoffMA. Infantile cataract in the collaborative perinatal project: prevalence and risk factors. Arch Ophthalmol. 2002;120(11):1559-65.

5. Wilson ME, Pandey SK, Thakur J. Paediatric cataract blindness in the developing world: surgical techniques and intraocular lenses in the new millennium. $\mathrm{Br} J$ Ophthalmol. 2003:87(1):14-9.

6. Foster A. Gilbert C, Rahi J. Epidemiology of cataract in childhood: a global perspective. J Cataract Refract Surg. 1997;23 Suppl 1:601-4.

7. Von Noorden GK, Campos EC. Binocular vision and ocular motility: theory and management of strabismus. $6^{\text {th }}$ ed. St. Louis: Mosby; 2002.

8. Muñoz B, West SK. Blindness and visual impairment in the Americas and the Caribbean. Br J Ophthalmol. 2002;86(5):498-504.

9. Gilbert C, Foster A. Childhood blindness in the context of VISION 2020-the right to sight. Bull World Health Organ. 2001;79(3):227-32.

10. Perucho-Martínez S, De-la-Cruz-Bertolo J, Tejada-Palacios P. Cataratas pediátricas: estudio epidemiológico y diagnóstico. Análisis retrospectivo de 79 casos. Arch Soc Esp Oftalmol. 2007;82(1):37-42.

11. González Viejo I, Ferrer Novella C, Pueyo Subias M, Melcón Sánchez B, Cuevas R, Bada T, Honrubia Lopez FM. Cataratas congénitas y adquiridas infantiles en nuestro medio. Arch Soc Esp Oftalmol. 1999;74:627-30.

12. Haargard B, Wohlfarht J, Rosemberg T, Fledelius HC, Melbye M. Risk Factors for idiopatic Congenital/Infantile Cataract. Invest Ophthalmol Vis Sci. 2005:46(9):3067-73.

13. Rahi JS, Dezateux C. Congenital and infantile cataract in the United Kingdom: underlying or associated factors. British Congenital Cataract Interest Group. Invest Ophthalmol Vis Sci. 2000;41(8):2108-14.

14. Wirth MG, Russell-Eggitt IM, Craig JE, Elder JE, Mackey DA. Aetiology of congenital and paediatric cataract in an Australian population. Br J Ophthalmol. 2002;86(7):782-6.

15. Chak M, Wade A, Rahi JS; British Congenital Cataract Interest Group. Long-term visual acuity and its predictors after surgery for congenital cataract: findings of the British congenital cataract study. Invest Ophthalmol Vis Sci. 2006;47(10):4262-9.

16. Yorston D, Wood M, Foster A. Results of cataract surgery in young children in east Africa. Br J Ophthalmol. 2001;85(3):267-71

17. Magnusson G, Jakobsson P, Kugelberg U, Lundvall A, Maly E, Tornqvist K, Abrahamsson $M$, et al. Evaluation of screening procedures for congenital cataracts. Acta Paediatr. 2003;92(12):1468-73.

18. Ye HH, Deng DM, Qian YY, Lin Z, Chen WR. Long-term visual outcome of dense bilateral congenital cataract. Chin Med J (Engl). 2007;120(17):1494-7. 